\title{
Eficácia de Ovitrampas com Diferentes Atrativos na Vigilância e Controle de Aedes
}

\author{
Priscila Aparecida Claro Depoli¹, João Antonio Cyrino Zequi2 ${ }^{\bowtie}$, \\ Kauani Larissa Campana Nascimento² \& José Lopes ${ }^{2}$
}

1. Universidade Estadual de Londrina, e-mail: priscila_depolli@hotmail.com, kauaninascimento@yahoo.com.br, jea@uel.br. 2. Instituto Nacional de Pesquisas da Amazônia (INPA/MCTI), e-mail: joaozequi@gmail.com (Autor para correspondência ${ }^{\varpi}$ ).

\section{EntomoBrasilis 9 (1): 51-54 (2016)}

Resumo. Aedes aegypti (Linnaeus) é o vetor dos vírus dengue, febre amarela urbana e Zika vírus nas Américas. Aedes albopictus (Skuse) tem maior ocorrência em mata nativa e secundária junto às populações. Esses mosquitos veiculam também o vírus chikungunya. $\mathrm{O}$ objetivo desse estudo foi avaliar a eficácia e preferência de oviposição em armadilhas ovitrampas, instaladas em locais abrigados com diferentes misturas atrativas, sob condições distintas de luminosidade e fluxo de pessoas para ser usada na vigilância e controle de Aedes spp. Foram instaladas 75 armadilhas no campus da Universidade Estadual de Londrina Paraná, distribuídas em três centros de estudo: Centro de Ciências Biológicas (CCB), Biblioteca Central (BC) e Centro de Estudos Sociais Aplicados (CESA), que receberam cada um, 25 ovitrampas, no período de Março a Junho de 2012. As armadilhas receberam cinco diferentes misturas atrativas: infusão de água fenada (Iaf), água destilada, (Iaf) + Bacillus thuringiensis var. israelensis Berliner, (Iaf) + organofosforado Temephos, (Iaf) + regulador de crescimento Diflubenzuron. Todo o experimento foi realizado em 5 réplicas. Coletou-se 4.774 ovos. No (CCB) foram 436 ovos (9,23\%) com Índice de Positividade de Ovitrampas (IPO) = 43,08\% e Índice de Densidade de Ovos (IDO) = 15,75. No (CESA) 638 ovos (13,35\%), IPO = 58,46\% e IDO = 16,79. Na (BC) 3.700 ovos $(77,42 \%)$, IPO = 83,08\% e IDO = 68,51. Não ocorreram diferenças significativas pelo teste de Kruskal-Wallis $(\mathrm{p}=0,92)$ para os diferentes atrativos nas ovitrampas. Atrativos e produtos seletivos para o controle de Aedes spp. na ovitrampa não mostraram prejuízos para oviposição.

Palavras-chave: Aedes albopictus; armadilha de sequestro de ovos; Bacillus thuringiensis israelensis; organofosforado; regulador de crescimento.

\section{Effectiveness of Ovitraps with Different Attractives in the Monitoring and Control of Aedes}

Abstract. Aedes aegypti (Linnaeus) is the vector of dengue viruses, urban yellow fever and Zika virus in the Americas. Aedes albopictus (Skuse) has higher incidence in native and secondary forest next the populations. These mosquitoes also convey the chikungunya virus. The aim of this study was to evaluate the efficacy and oviposition preference in ovitraps traps installed in sheltered places with different attractive mixtures, under different lighting conditions and flow of people to be used in monitoring and control of Aedes spp. Were installed 75 traps on campus of the State University of Londrina-Paraná, distributed in three study centers: Centre for Biological Sciences (CCB), Central Library (CL) and Applied Social Studies Center (ASSC), which each received 25 ovitraps in the period from March to June 2012. The traps received five different attractive mixtures: infusion of water made into hay (Iaf), distilled water (Iaf) + Bacillus thuringiensis israelensis Berliner (Iaf) + organophosphate temephos, (Iaf) + growth regulator Diflubenzuron. The experiment was carried out in 5 replicates. Collected: 4,774 eggs. On (CCB) were 436 eggs (9.23\%) with Positivity Index of Ovitraps $(\mathrm{PIO})=43.08 \%$, and Eggs Density Index $(\mathrm{EDI})=15.75$. On $(\mathrm{ASSC}) 638$ eggs $(13.35 \%)$, PIO $=58.46 \%$ and EDI $=16.79$. On $(\mathrm{CL}) 3,700$ eggs $(77.42 \%)$, PIO $=83.08 \%$ and EDI $=68.51$. There were no significant differences by Kruskal-Wallis test $(\mathrm{p}=0.92)$ for the different attractive on ovitraps. Attractive and selective products for Aedes spp. control in egg trap do not show damage to oviposition.

Keywords: Aedes albopictus; Bacillus thuringiensis israelensis; growth regulator; kidnapping trap eggs; organophosphate.

edes (Stegomyia) aegypti (Linnaeus) (Diptera: Culicidae) é o vetor do dengue e da febre amarela urbana no Brasil (ForatTini 2002). Recentemente veicula também o Zika vírus. A dengue é a arbovirose mais importante do mundo com aumento de 30 vezes no número de casos registrados anualmente nos últimos 50 anos, expondo quase metade da população global ao risco a esta infecção (WHO 2014). Cinquenta a 100 milhões de casos da doença são estimados por ano em mais de 100 países endêmicos, se caracterizando como enfermidade que mais causa hospitalização e morte em crianças na Ásia e América Latina (WHO 2014).

Aedes albopictus (Skuse) (Diptera: Culicidae) é conhecido como tigre asiático. As fêmeas colocam seus ovos na parte interna de recipientes diversos, tanto de origem antrópica como naturais nas áreas urbanas, suburbanas, rurais, e também próximo de áreas florestais. O mosquito é abundante em áreas com vegetação próximas a residências (ForATTini 2002). Desde seu encontro no Brasil em 1986 (ForatTinI 1986), não tem sido incriminado por epidemias de dengue no país, mas se infecta naturalmente com o vírus da febre amarela e da dengue, além de ter potencial em laboratório para se infectar com mais de 20 arbovírus (Moore \& Mitchell 1997). Para o vírus Chikungunya, Ae. aegypti e Ae. albopictus, são eficientes vetores no Brasil, e Ae. albopictus é hábil para veicular o patógeno em apenas dois dias após a infecção em laboratório (VEGA-RÚA et al. 2014).

As precárias condições de saneamento básico, a inexistência de um controle adequado dos mosquitos e a carência de programas de Saúde Púbica favorecem esses vetores e a disseminação viral (CAmpanelli 2007).

Estratégias para monitoramento e controle desses insetos são necessárias para amenizar a circulação viral e garantir qualidade de vida à população. O uso de ovitrampas é uma importante ferramenta para monitorar e controlar populações destes mosquitos (REGIS et al. 2008). Melhorar e adequar armadilhas de oviposição são estratégias para otimizar o monitoramento e controle desses importantes vetores sinantrópicos (Acioli 2006). A utilização de ovitrampas configura-se como um método apropriado de detecção da presença e a verificação da densidade populacional de A. aegypti em diferentes ambientes (VEZZANi et al. 
T200 2004; Rios-VELÁsqueZ et al. 2007; RUEDA 2009). Esta metodologia também tem sido defendida junto ao controle integrado desses vetores e detecções de infestações, por (Marques et al. 1993; Morato et al. 2005, Gama et al. 2007; Regis et al. 2008).

O objetivo desse estudo foi avaliar a eficácia e preferência de oviposição em armadilhas ovitrampas, instaladas em locais abrigados com diferentes misturas atrativas, sob condições distintas de luminosidade e fluxo de pessoas para ser usada na vigilância e em estratégias de controle de Aedes spp.

\section{MATERIAL E MÉTODOS}

Estudo realizado entre março a junho de 2012. A área de estudo foi o campus da Universidade Estadual de Londrina, localizada na Rodovia Celso Garcia Cid (PR-445), Km 380. O campus universitário possui 235,57 hectares. Determinaram-se três locais equidistantes para instalação das ovitrampas: Centro de Ciências Biológicas (CCB), Biblioteca Central (BC) e Centro de Ciências Sociais Aplicadas (CESA) (Figura 1). O CCB e a BC estão circundadas por outros prédios, com grande fluxo de pessoas e o CESA, caracteriza-se como área limítrofe entre os prédios do campus e ambiente de fragmento florestal, devido a suas proximidades com o bosque da universidade. Neste centro também há grande circulação de pessoas.

As armadilhas de oviposição, também conhecidas por ovitrampas, ovitraps ou armadilhas de sequestro de ovos, utilizadas na obtenção de ovos de Aedes spp. são vasos plásticos de coloração preta com capacidade de $500 \mathrm{~mL}$. No seu interior adicionaram-se $300 \mathrm{~mL}$ da mistura atrativa. O fluido padrão esteve constituído por $250 \mathrm{~mL}$ de água destilada e $50 \mathrm{~mL}$ de água fenada obtidas através da adição de $34 \mathrm{~g}$ de capim Colonião [Megathyrsus maximus (Jacq.)] em $8 \mathrm{~L}$ de água, fermentado por cinco dias em balde plástico na temperatura ambiente. No interior das armadilhas introduziu-se um filete de Duratree de $15 \times 3 \mathrm{~cm}$.

Utilizaram-se cinco misturas para atração das fêmeas nas armadilhas de oviposição; somente água destilada (controle) - (Ad); água destilada com acréscimo de água fenada (Iaf); Iaf acrescida de $1 \mathrm{mg} / \mathrm{L}$ de Bacillus thuringiensis var. israelensis Berliner (IBti); Iaf acrescida de $200 \mathrm{mg} / \mathrm{L}$ do Organofosforado Temephos (Ifof); Iaf mais $300 \mathrm{mg} / \mathrm{L}$ de regulador de crescimento Diflubenzuron (Irc). As concentrações do bioinseticida, regulador de crescimento e organofosforado representam a concentração indicada pelo Ministério da Saúde para controle de larvas de Aedes spp. (FUNASA 2001; SVS 2005).

Foram instaladas 75 armadilhas, 25 em cada área de estudo em três réplicas, dentro das salas dos respectivos centros, locais não climatizados e posicionadas no solo, nos cantos de paredes, abaixo de mesas e/ou mobiliário com as seguintes condições:

a) penumbra: (ambiente que recebe luminosidade artificial em horário comercial das 8 às 18 horas); mais os cantos de parede, locais abaixo de mesas, que são de penumbra;

b) escuro (ambiente constantemente sem iluminação natural ou artificial);

c) com permanência de pessoas (ambiente com permanência de pessoas das 8 às 18 horas);

d) com fluxo contínuo de pessoas (corredores de acesso às salas);

e) isolados (depósitos de armazenamento de materiais).

Consideraram-se as combinações (penumbra com ou sem fluxo de pessoas, e local isolado e escuro) para o cálculo do Índice de Densidade Vetorial que é uma relação do total de ovos coletados por ovitrampas instaladas e pode inferir sobre a densidade ou atividade do vetor (AvENDANHA 2007).

Semanalmente, durante 05 repetições, as palhetas eram recolhidas para a quantificação dos ovos e uma nova reinstalada. Com aparelho Multiparâmetro (Hanna HI 9828) analisava-se quinzenalmente amostras da mistura atrativa das armadilhas, para mensurar o $\mathrm{pH}$, salinidade, condutividade e temperatura da água no momento da amostragem desses dados.

Realizou-se estatística descritiva com médias e desvios padrão. A normalidade foi verificada com o teste Shapiro-Wilk e empregado o teste não paramétrico de Kruskal-Wallis a $5 \%$ de significância, para avaliar a preferência de oviposição de Aedes spp. em ovitrampas contendo os diferentes atrativos, além de verificar a preferência de oviposição em armadilhas distribuídas nas diferentes condições de fluxo de pessoas. Para preferência de oviposição quanto à luminosidade utilizou-se o teste não paramétrico de Mann-Whitney com programa Past (PAST version 2.15) (Hammer et al. 2001). Para comparar Índices de Densidade Vetorial nos diferentes Centros, utilizou-se o teste de Tukey no nível de $5 \%$ com o pacote SPSS ${ }^{\circledR} 14.0$ package for Windows.

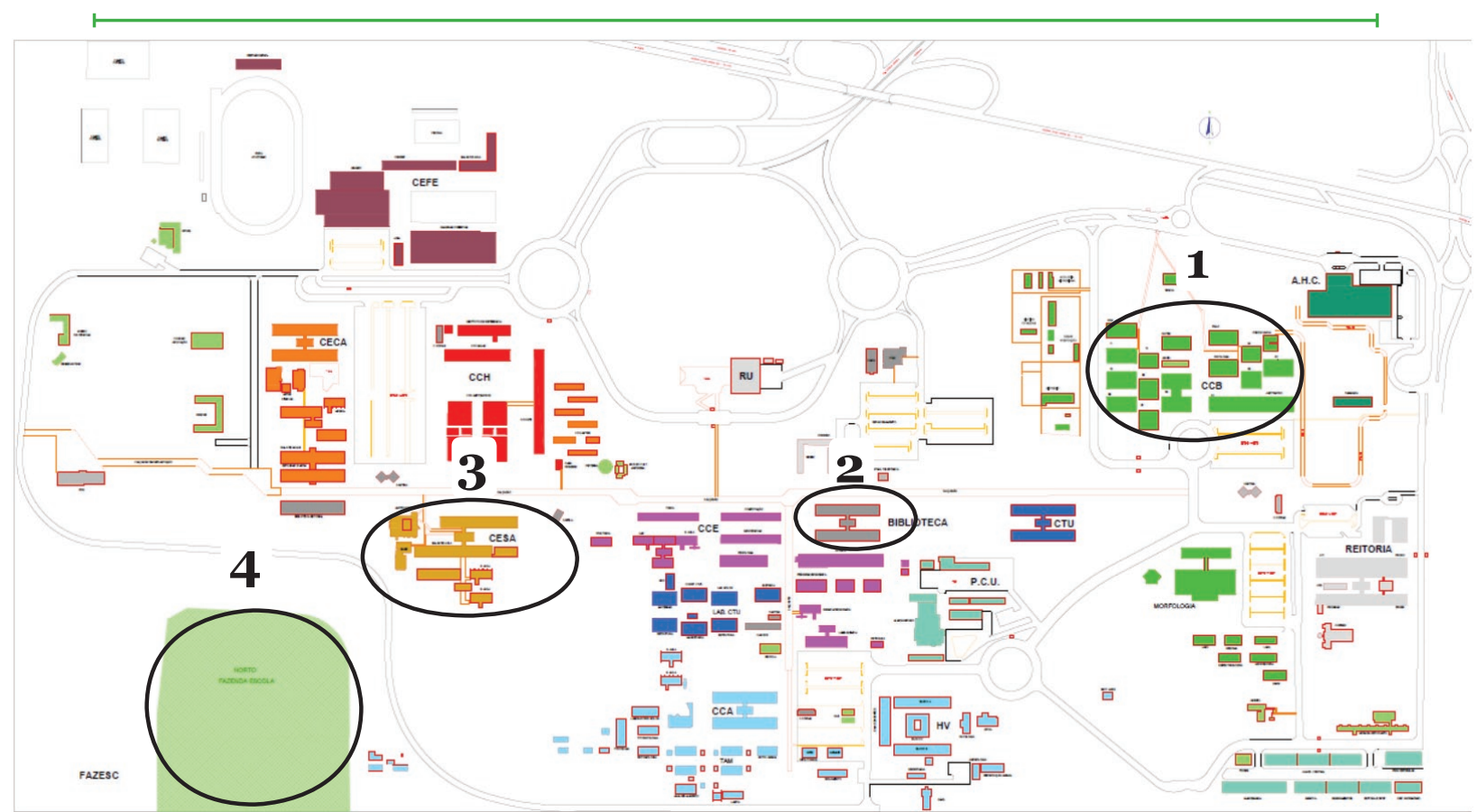

Figura 1. Mapa do Campus da Universidade Estadual de Londrina (UEL), indicando os centros de estudo onde foi implantado armadilhas ovitrampa, no período de Março a Junho de 2012, Londrina - Paraná - Brasil. 1- CCB = Centro de Ciências Biológicas; 2- BC = Biblioteca Central; 3- CESA = Centro de Ciências Sociais Aplicadas; 4- FAZESC = Horto Fazenda Escola. Fonte: Pró - Reitoria de Planejamento/Diretoria de Planejamento e Desenvolvimento Físico (PROPLAN/DPDF). 


\section{RESULTADOS E DISCUSSÃO}

Foram coletados 4.774 ovos (Figura 2). No Centro de Ciências Biológicas (CCB) coletou-se 436 ovos (9,23\%) com Índice de Positividade de Ovitrampas (IPO) $=43,08 \%$, Índice de Densidade de Ovos (IDO) $=15,75$ e Índice de Densidade Vetorial (IDV) $=$ $1,45^{\mathrm{b}}$. No Centro de Estudos Sociais Aplicados (CESA), 638 ovos $(13,35 \%)$, IPO $=58,46 \%$, IDO $=16,79$ e IDV $=2,13^{\mathrm{b}} . \quad \mathrm{Na}$ Biblioteca Central obteve-se 3.700 ovos $(77,42 \%)$, IPO = 83,08\%, $\mathrm{IDO}=68,51 \mathrm{e}$ IDV $=12,33^{\mathrm{a}}$. Há diferença estatística pelo teste de Tukey ( $\mathrm{p}<0,1 \%$, onde ${ }^{\mathrm{a}} \mathrm{e}^{\mathrm{b}}$ diferem significativamente).

Dois fatores principais podem ter influenciado na maior quantidade de ovos de Aedes spp. na Biblioteca Central. Neste local ocorrem as maiores circulações e permanência de pessoas nos períodos diurno, vespertino e noturno. No entorno do edifício havia recipientes de materiais de construção propícios ao acúmulo de água.

Armadilhas contidas em ambientes de penumbra totalizaram $68,74 \%$ dos ovos coletados, completando com $31,26 \%$ em local escuro. Mesmo observando-se disparidade entre os valores encontrados, não há diferença estatística $(\mathrm{p}=0,3827)$, pelo teste de Mann-Whitney. Também pelo teste de Kruskall-Wallis não ocorrem diferenças estatísticas nos resultados obtidos com armadilhas instaladas em locais com fluxo de pessoas e aquelas localizadas onde não havia acesso regular de funcionários. Locais com permanência de pessoas representaram $(64,28 \%)$ de posturas, fluxo de pessoas $(32,39 \%)$ e local isolado $(3,33 \%)$, ( $\mathrm{p}=0,2881$ ). Estes dados estão de acordo com os obtidos por NunEs et al. (2011), que constataram, em armadilhas instaladas próxima ao centro da cidade, maior ocorrência de ovos, havendo uma correlação com maior concentração de pessoas. Resultado semelhante foi obtido por BARATA et al. (2001), que relatou locais com alta concentração de pessoas, e maior registro de $A e$. aegypti. A ausência de diferença nos resultados obtidos para as armadilhas instaladas onde havia fluxo de pessoas com os ambientes isolados, pode estar relacionada com a proximidade de onde foram instaladas as armadilhas, pois estavam distante entre elas no mínimo $20 \mathrm{~m}$. Ressalta-se também que o encontro de maior quantidade de ovos em locais de penumbra pode estar relacionado à presença e/ou permanência de pessoas de forma constante, onde os insetos poderiam fazer o repasto sanguíneo, se abrigar em microambientes sombreados e posteriormente realizar postura nas ovitrampas instaladas em cantos de paredes ou partes inferiores de mobiliário.

Para locais com penumbra com ou sem fluxo de pessoas (PCSFP) e Local isolado e escuro (IE) obteve-se respectivamente os seguintes Índices de Densidade Vetorial: CCB (PCSFP $=1,2$ e IE $=4,38) ; \mathrm{BC}(\mathrm{PCSFP}=13,21 \mathrm{e} \mathrm{IE}=2,17)$ e CESA $(\mathrm{PCSFP}=2,27 \mathrm{e}$ $\mathrm{IE}=1,08)$.

Mesmo ocorrendo reduções no IDV nas duas últimas semanas de coletas em todos os locais estudados não há diferença significativa ( $\mathrm{p}>5 \%$ ) de redução de ovos nas ovitrampas ao longo do experimento.

Obtiveram-se os seguintes resultados quanto aos parâmetros físicos químicos nas misturas atrativas no ato da instalação da armadilha: pH médio 6,85 (5,68 - 6,85); salinidade 0,07 mg/L (o mg/L - o,15 mg/L); temperatura do criadouro $25,33{ }^{\circ} \mathrm{C}(21,04$ $\left.{ }^{\circ} \mathrm{C} .-27,13^{\circ} \mathrm{C}\right)$ e condutividade $91,18 \mu \mathrm{s} / \mathrm{cm}(1,64 \mu \mathrm{s} / \mathrm{cm}-170 \mu \mathrm{s} /$ $\mathrm{cm})$. Após quinze dias os resultados foram: $\mathrm{pH}$ médio $=7,24(6,27$ - 7,37); salinidade $0,05 \mathrm{mg} / \mathrm{L}$ (o mg/L - 0,08mg/L); temperatura nas ovitrampas $25,39{ }^{\circ} \mathrm{C}\left(21,1{ }^{\circ} \mathrm{C}-27,7{ }^{\circ} \mathrm{C}\right)$ e condutividade $104,5 \mu \mathrm{s} / \mathrm{cm}(7 \mu \mathrm{s} / \mathrm{cm}-176 \mu \mathrm{s} / \mathrm{cm})$. Conclui-se que os produtos para controle de larvas introduzidos nas ovitrampas não afetam os padrões físicos e químicos da amostra.

Na Tabela 1 são relacionados o Índice de Densidade de Ovos (IDO) e o Índice de Positividade de Ovitrampas (IPO) nos diferentes ambientes e tratamentos utilizados.

Para as fêmeas de culicídeos localizarem e optarem pelo local para a oviposição há envolvimento de uma série de fatores; reações visuais, olfativas e táteis (CosTa 2010). Quando inserido infusão de capim colonião M. maximus em armadilhas de oviposição, esse

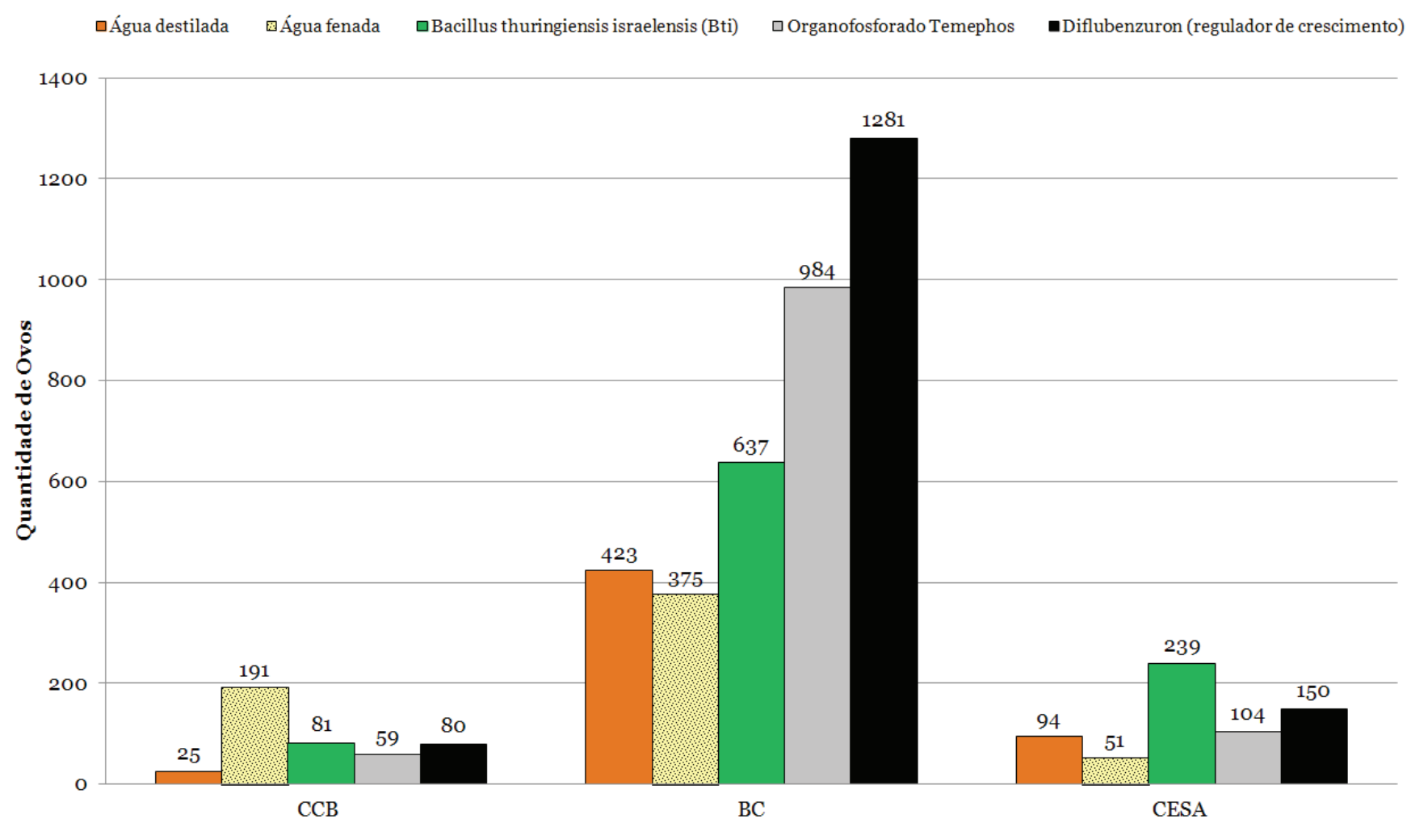

Atrativos

Figura 2. Total de ovos de Aedes spp. em ovitrampas com diferentes misturas atrativas nos centros de estudo da UEL, Londrina, Paraná, no período de Março a Junho de 2012. CCB = Centro de Ciências Biológicas, BC = Biblioteca Central, CESA = Centro de Ciências Sociais Aplicadas. 
Tabela 1. Índice de Positividade de Ovitrampas (IPO) e Índice de Densidade de Ovos (IDO) de acordo com os tratamentos e infusões em ovitrampas instaladas nos Centros de Ciências Biológicas (CCB), Biblioteca Central (BC) e Centro de Estudos Sociais Aplicados (CESA).

\begin{tabular}{|c|c|c|c|c|c|c|}
\hline \multirow{2}{*}{ Atrativos (infusões) } & \multicolumn{3}{|c|}{ IPO } & \multicolumn{3}{|c|}{ IDO } \\
\hline & CCB & BC & CESA & CCB & BC & CESA \\
\hline Água destilada & $46,15 \%$ & $76,92 \%$ & $53,84 \%$ & 4,17 & 42,3 & 13,43 \\
\hline Água fenada (Iaf) & $46,15 \%$ & $76,92 \%$ & $38,46 \%$ & 31,83 & 37,5 & 10,2 \\
\hline Bacillus thuringiensis var. israelensis (Ibti) & $46,15 \%$ & $84,61 \%$ & $84,61 \%$ & 13,5 & 57,91 & 21,73 \\
\hline Organofosforado Temephos (Ifof) & $30,76 \%$ & $84,61 \%$ & $53,84 \%$ & 14,75 & 88,55 & 14,85 \\
\hline Infusão do Diflubenzuron (Irc) & $46,15 \%$ & $61,53 \%$ & $61,53 \%$ & 14,17 & 106,75 & 18,75 \\
\hline
\end{tabular}

fermentado torna-se um atrativo estimulante, sendo perceptíveis pelas fêmeas a longas distâncias (BENTLEY \& DAY 1989), sendo considerado um importante atrativo para a fêmea de Aedes spp. (SANT'ANA et al. 2006).

Mesmo utilizando a infusão de capim, a oviposição nas armadilhas com as diferentes misturas, nos diferentes sítios de estudo, não divergiram estatisticamente por Kruskal-Wallis $(p=0,92)$, em relação a quantidade de ovos (Figura 2). Este resultado significa que adicionando (Bti), Temephos ou Diflubenzuron a água fenada ou destilada, não há alteração na preferência de oviposição pelas fêmeas.

NAScimento (2013) relacionou estudos com ovitrampas concomitante com os levantamentos larvários (LIRAa), em diferentes localidades no município de Cambé-Paraná, observando a presença e a densidade de ovos de Ae. aegypti em ovitrampas, enquanto que o método de levantamento larvário não detectou a presença do vetor.

Segundo Acioli (2006), através de levantamento feito por meio das ovitrampas torna-se possível estimar a população de fêmeas de Ae. aegypti em atividade reprodutiva atual e promover avaliação contínua da população do mosquito com eficaz controle populacional.

A utilização do controle biológico e/ou produtos seletivos para imaturos em conjunto com a ovitrampa tem a vantagem de minimizar os danos ambientais que os inseticidas químicos podem causar. O B. thuringiensis var. israelensis (Bti), apresenta boa atividade contra larvas de Aedes spp. Este estudo mostrou que a presença deste produto na água do criadouro não repele a fêmea grávida, o que garante sua eficácia quando utilizado nos programas de controle de Ae. aegypti, podendo assim ser utilizado em armadilhas para atração da fêmea e ao mesmo tempo impedir o desenvolvimento larvário, em possível eclosão do ovo.

SToops (2005) constatou maior frequência de oviposição para Ae. albopictus com utilização de B. thuringiensis var. israelensis (Bti) em relação ao controle.

Conforme as Diretrizes Nacionais para a Prevenção e Controle da Dengue estabelecida pelo Ministério da Saúde do Brasil em 2009, bioinseticidas que tenham como principio ativo o $B$. thuringiensis var. israelensis, devem ser utilizado de maneira rotativa com o organofosforado Temephos, para evitar a seleção de larvas resistentes ao larvicida químico. Atualmente o Ministério da Saúde tem autorizado a utilização dos reguladores de crescimento, em especial inseticidas inibidores da síntese de quitina Diflubenzuron e Novaluron (ProphIro et al. 2011; Flores et al. 2013), nas ações de controle, principalmente nos estados onde as larvas do mosquito têm se mostrado resistente ao Temephos.

Os resultados obtidos na presente pesquisa conciliam e otimizam a utilização de produtos alternativos como regulador de crescimento e bioinseticida com uso de ovitrampas para o monitoramento e estratégias de controle de Ae. aegypti e Ae. albopictus na área urbana e periurbana, mas estudos mais extensivos são necessários para validar a metodologia.
Com rigoroso monitoramento, as armadilhas ovitrampas, consorciadas a produtos de controlelarval seletivo também poderá ser utilizada para o controle do mosquito, já que sequestram os ovos e não permitem o desenvolvimento larvário caso ocorram eclosões dos ovos. A utilização de Diflubenzuron (regulador de crescimento) e B. thuringiensis var. israelensis (Vectobac 12AS) pelo fato de não repelirem as fêmeas de Aedes spp. no processo de oviposição e não alterarem as condições físico-química da água, como o pH, salinidade, e condutividade, são produtos viáveis, aumentando inclusive o intervalo de inspeção às ovitrampas, reduzindo assim a logística com pessoal para monitoramento semanal.

Pela quantidade de ovos obtidos neste estudo por meio das ovitrampas, e os respectivos IPO e IDO (Tabela 1), verificouse sua eficiência no monitoramento de Aedes aegypti e Ae. albopictus, em área urbana. Estudos com amostragens maiores em diferentes regiões poderão consolidar a metodologia para incremento nos programas oficiais do governo como estratégia de monitoramento para controle de dengue, chikungunya e Zika vírus.

O baixo custo dessa metodologia e a sua sensibilidade em demonstrar a presença de mosquitos na região são condições importantes para tornar essa armadilha mais uma ferramenta de vigilância.

Embora o uso de água fenada nas armadilhas é um padrão como atrativo (SANT'ANA et al. 2006), a água sem qualquer substância mostrou-se eficiente, sem significativa perda na atratividade de Aedes spp. (Tabela 1). Esta indicação pode facilitar ainda mais a utilização e manipulação destas armadilhas.

\section{AGRADECIMENTOS}

Ao Laboratório de Entomologia Médica e Geral da Universidade Estadual de Londrina, onde foi desenvolvido todo o trabalho.

\section{REFERÊNCIAS}

Acioli, R.V., 2006. O uso de armadilhas de oviposição (ovitrampas) como ferramenta para monitoramento populacional do Aedes spp. em bairros do Recife. Dissertação de Mestrado em Saúde Pública. Fundação Oswaldo Cruz "Centro de Pesquisa Aggeu Magalhães", Recife, 13op.

Avendanha, J.S., 2007. Monitoramento vetorial e do vírus Dengue, Belo Horizonte, Minas Gerais. Revista do Instituto Adolfo Lutz, [S.1.], 66: 207-207. Disponível em: $<$ http://revistas.bvs-vet.org.br/rialutz/article/view/7768>. [Acesso em: 15.04.2015].

Barata, E.A.M.F., A.I.P. Costa, F. Chiaravalloti Neto, C.M. Glasser, J.M.S. Barata \& D. Natal, 2001. População de Aedes aegypti (I.) em área endêmica de dengue, Sudeste do Brasil. Revista Saúde Pública, 35: 237-242.

Bentley, M.D. \& J.F. Day, 1989. Chemical Ecology and Behavioral Aspects of Mosquito Oviposition. Annual Review of Entomology, 34: 401-421.

Campanelli, E.S., 2007. O desenvolvimento de um processo de infecção do Aedes aegypti pelo vírus dengue: caracterização da interação do vírus com uma população de mosquitos 
autóctones. Dissertação (Mestrado m Ciências da Saúde). Fundação Oswaldo Cruz "Centro de Pesquisas René Rachou”, Belo Horizonte, 91p.

Costa, L.H., 2010. Avaliação de infoquímicos presentes em ovos e larvas de Aedes (Stegomyia) aegypti (Linnaeus, 1762) (Diptera: Culicidae) como atraentes e/ou estimulantes de oviposição para grávidas co-específicas. (Dissertação Mestrado em Parasitologia). Universidade Federal de Minas Gerais. Minas Gerais, 128 p.

Flores, A.E., G. Ponce, B.G. Silva, S.M. Gutierrez, C. Bobadilla, B. Lopez, R. Mercado \& W.C. Black, 2013. Wide Spread Cross Resistance to Pyrethroids in Aedes aegypti (Diptera: Culicidae) from Veracruz State Mexico. Journal of Economic Entomology, 106: 959-969.

Forattini, O.P., 1986. Identification of Aedes (Stegomyia) albopictus (Skuse) in Brazil. Revista de Saúde Pública, 20: 244-245.

Forattini, O.P., 2002. Culicidologia médica: Identificação, Biologia, Epidemiologia. Aedes (Stegomyia). $1^{\text {a }}$ ed, vol. 2, São Paulo, EDUSP, 506p.

FUNASA, 2001. Dengue - Instruções para pessoal de combate ao vetor: Manual de normas técnicas. Fundação Nacional de Saúde. Disponível em: <http://bvsms.saude.gov.br/ bvs/publicacoes/funasa/man dengue.pdf $>$. [Acesso em: 13.04.2015].

Gama, R.A., A.E. Eiras \& M.C. Resende, 2007. Efeito da ovitrampa letal na longevidade de fêmeas de Aedes aegypti (Diptera: Culicidae). Revista da Sociedade Brasileira de Medicina Tropical, 40: 640-642.

Hammer, Ø., D.A.T. Harper \& P.D. Ryan 2001. PAST: Paleontological statistics software package for education and data analysis. Palaeontologia Electronica, 4: 9 p.

Marques, C.C.A., G.R.A.M. Marques, M. Brito, L.G.S. Neto, V.C. Ishibashi \& F.A. Gomes, 1993. Estudo comparativo de eficácia de larvitrampas e ovitrampas para vigilância de vetores de dengue e febre amarela. Revista Saúde de Pública, 27: 237241.

Moore, C.G. \& C.J. Mitchell, 1997. Aedes albopictus in the United States: ten-year presence and public health implications. Journal Emerging Infectious Diseases, 3: 329-334.

Morato, V.C.G., M.G. Teixeira, A.C. Gomes, D.P. Bergamaschi \& M. Barreto, 2005. Infestation of Aedes aegypti estimated by oviposition traps in Brazil. Revista de Saúde Pública, 39: 553558 p.

Nascimento, K.L.C., 2013. Avaliação de eficiência no uso de armadilhas de oviposição para diagnóstico e estimativa de população de Aedes (Stegomyia) aegypti (Linnaeus, 1762). Dissertação (Mestrado em Ciências Biológicas). Universidade Estadual de Londrina. Londrina, $91 \mathrm{p}$.

Nunes, L.S., R.B.R. Trindade \& R.N.P. Souto, 2011. Avaliação da atratividade de ovitrampas a Aedes (Stegomyia) aegypti Linneus (Diptera: Culicidae) no bairro Hospitalidade, Santana, Amapá. Biota Amazônia Open Journal System, 1: 26-31.
Prophiro, J.S., O.S. Silva, J.E. Luna, C.F. Piccoli, L.A. Kanis \& M.A. Silva, 2011. Aedes aegypti and Aedes albopictus (Diptera: Culicidae): coexistence and susceptibility to temephos, in municipalities with occurrence of dengue and differentiated characteristics of urbanization. Revista da Sociedade Brasileira de Medicina Tropical, 44: 300-305.

Regis, L., A.M. Monteiro, M.A.V. Melo-Santos, J.C. Silveira Jr, A.F. Furtado, R.V. Acioli, G.M. Santos, M. Nakazawa, M.S. Carvalho, P.J. Ribeiro Jr \& W.V. Souza, 2008. Developing new approaches for detecting and preventing Aedes aegypti population outbreaks: basis for surveillance, alert and control system. Memórias do Instituto Oswaldo Cruz, 103: 50-59.

Rios-Velásquez, C.M., C.T. Codeço, N.A. Honório, P.S. Sabroza, M. Moresco, I.C.L. Cunha, A. Levino, L.M. Toledo \& S.L.B. Luz, 2007. Distribution of dengue vectors in neighborhoods with different urbanization types of Manaus, state of Amazonas, Brazil. Revista Memórias do Instituto Oswaldo Cruz, 102: 617-623.

Rueda, B.Z., 2009. Comparação da eficácia dos métodos "Índice de Breteau" e armadilha de oviposição (ovitrampas) na obtenção dos índices de infestação de Aedes (Stegomyia) aegypti e Aedes (Stegomyia) albopictus no Município de Botucatu, SP. Dissertação (Mestrado em Biologia Geral). Universidade Estadual Paulista, Instituto de Biociências, Botucatu, $67 \mathrm{p}$

Sant'Ana, A.L., R.A. Roque \& A.E. Eiras, 2006. Characteristics of grass infusion as oviposition attractants to Aedes (Stegomyia) (Diptera: Culicidae). Journal of Medical Entomology, 43: 214-220.

Stoops, C.A., 2005. Influence of Bacillus thuringiensis var. israelensis on oviposition of Aedes albopictus (Skuse). Journal of Vector Ecology, 30: 41-44.

SVS, 2005. Avaliação da eficácia de análogos de hormônio juvenil e inibidores da síntese de quitina no controle de Aedes aegypti. Secretaria de Vigilância em Saúde. Disponível em: <http:// bvsms.saude.gov.br/bvs/publicacoes/avaliacao eficacia analogos aedes aegypti.pdf $>$. [Acesso em: 13.04.2015].

Vega-Rúa, A., K. Zouache, R. Girod, A.B. Failloux \& R. Lourenço de Oliveira, 2014. High vector competence of Aedes aegypti and Aedes albopictus from Ten American Countries as a crucial factor of the spread of Chikungunya virus. Journal of Virology. JVI.o0370-14, 88: 6294-6306.

Vezzani, D., S.M. Velázquez \& N. Schweigmann, 2004. Seasonal pattern of abundance of Aedes aegypti (Diptera: Culicidae) in Buenos Aires City, Argentina. Revista Memórias do Instituto Oswaldo Cruz, 99: 351-356.

WHO, 2014. Dengue. World Health Organization. Disponível em: < http://www.who.int/denguecontrol/en/index.html >. [Acesso em: 24.09.2014].

\section{Recebido em: 05.v.2015 \\ Aceito em: 03.ix.2015}

\section{Como citar este artigo:}

Depoli, P.A.C., J.A.C. Zequi, K.L.C. Nascimento \& J. Lopes, 2016. Eficácia de Ovitrampas com Diferentes Atrativos na Vigilância e Controle de Aedes. EntomoBrasilis, 9 (1): 51-55.

Acessível em: doi:10.12741/ebrasilis.v9i1.509
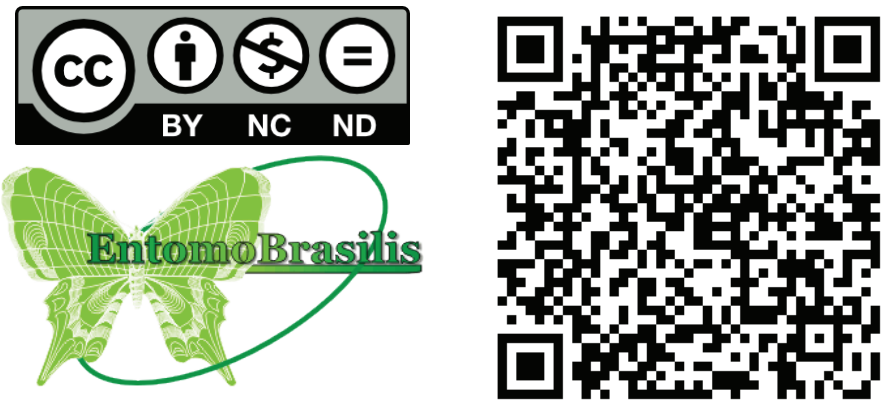